\title{
The Study of Femtosecond Laser Surface Textures Under Full Lubrication Conditions for Use in Liner
}

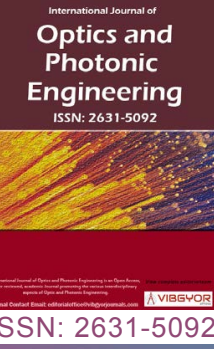

\section{Zhang Pan ${ }^{1}$, Chen Lei ${ }^{*}$ and Wei Haijun ${ }^{2}$}

\author{
${ }^{1}$ Logistic Engineering College, Shanghai Maritime University Shanghai, China \\ ${ }^{2}$ Merchant Marine College, Shanghai Maritime University, Shanghai, China
}

\begin{abstract}
Surface texturing in general and laser surface texturing (LST) in particular has emerged in recent years as a potential new technology to reduce friction in a diesel engine. In this paper, the mathematical model of the hydrodynamic pressure for different morphologies dimples are developed to analyze the relevant mechanism for the effect of surface texturing on reducing friction. The simulation results show that surface texturing is important for reducing friction and texturing surface with spherical cap dimple has the highest hydrodynamic pressure Microstructure of dimple for the sample surface is changed by LST, in order to change lubrication regime of surface. An experimental study was carried out to investigate the tribological performance of micro-dimple, for use in a liner. Compared to non-textured surfaces, the texturing surface with dimple shows significant tribological improvement.
\end{abstract}

\section{Keywords}

Laser surface texturing, Friction reduction, Femtosecond laser, Ablation depth, Micro-dimple

\section{Introduction}

It is so critical that the tribological properties of mechanical systems to improve their load carrying capacity and Service life. By reducing friction and wear has become an urgent need to improve efficiency and protective environment in the field of diesel engine $[1,2]$. About 40 percent of whole energy from a diesel engine is consumed by the engine friction [3]. Approximately $50 \%$ of all the friction losses in a diesel engine are due to the piston and cylinder system. Hence the efforts are from researchers of all over the world, to reduce the friction between the all kinds of moving parts in a diesel engine. Particularly the friction of the piston and cylinder liner was the focus of more study work.

The previous literatures [4-9] studied that the change and attribute of the film thickness between the piston and cylinder liner system based on the smooth surfaces. That hypothesis leads to do not match between the calculation results and test results. From the study results of a great deal of relevant literatures, the proper lubrication and surface texturing are key factors to reduce friction in a piston. Surface texturing, namely the processing of micro-dimple or mi-

*Corresponding author: Chen Lei, Merchant Marine College, Shanghai Maritime University, Shanghai, 201306, China

Accepted: October 10, 2019; Published: October 12, 2019

Copyright: ( 2019 Pan Z, et al. This is an open-access article distributed under the terms of the Creative Commons Attribution License, which permits unrestricted use, distribution, and reproduction in any medium, provided the original author and source are credited.

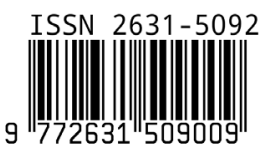

Pan et al. Int J Opt Photonic Eng 2019, 4:018 
cro-grooves of a certain size and arrangement is produced on the surface of the moving part, has proven to be an effective method to improve the tribological properties of the surface [10]. The principle of this way is to reduce the friction and improve surface bearing capacity which depends on the lubricating effect of fluid dynamic pressure that generated by the micro-groove or micro-dimple. The laser surface texturing (LST) as a useful means to enhance tribological properties of mechanical components in a diesel engine is received more attention for many years. The design idea of improving tribological properties based on the surface texturing was formed in the 1960s. Hamilton, et al. [11] proposed the idea to generate additional lubrication effects of dynamic pressure. Subsequently, most scholars have conducted a large number of theoretical and experimental studies. Various forms and geometric features of surface texturing for tribological applications are widely performed $[12,13]$. The literatures $[14,15]$ show that the structure of texturing can act as a micro-reservoir for lubricants, thereby reducing friction and extending the service life of moving surfaces. The optimized design of the surface texturing is an important part of the surface texturing technology. The study results indicate that the effect of surface texturing to reduce the friction was considerably depended on the geometric shape, size, distribution density, and pattern of dimples $[16,17]$ and the suitable selection of surface texturing parameters can achieve the optimal tribological properties of the texturing surface. Etsion [18] indicated that partial LST piston rings could consume up to $4 \%$ less fuel compared with non-textured conventional barrel-shaped rings. However, the study results from different researchers are not an agreement in terms of the choice of texturing geometry parameters. At present, there is no guiding surface texturing design method and theory yet. Therefore, the research in this field needs to be further strengthened.

\section{Analysis Model}

The aim in this paper is to study the basic theoretical research on the lubrication performance of micro-dimples. For the convenience of research, it is assumed that a plane friction pair consists of two surfaces in relative motion. The upper and lower contact surfaces are considered to constitute the non-textured surface and textured surface, respec-

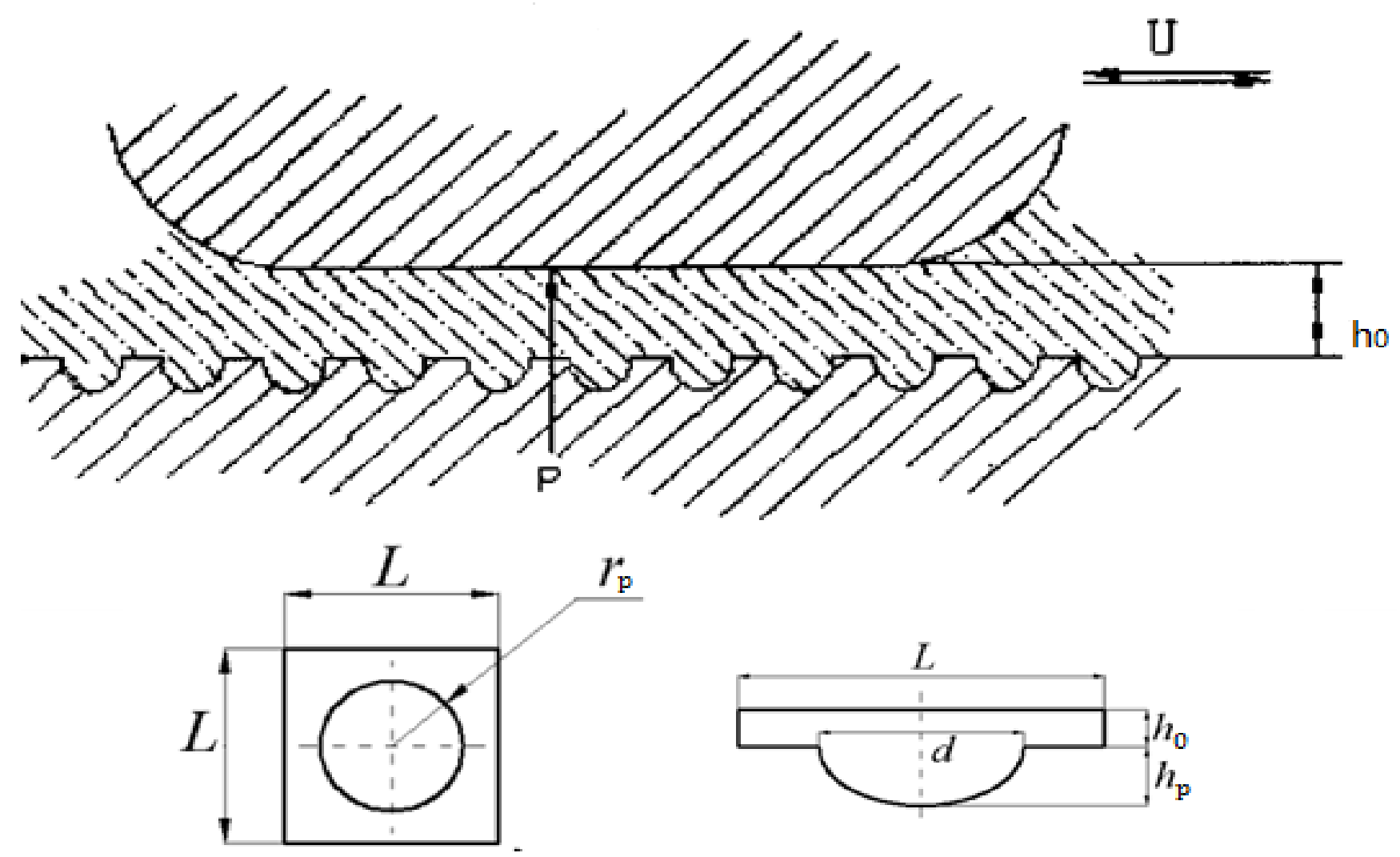

Figure 1: Schematic of two contacting surfaces with relative sliding velocity. 
tively, and are separated by a fluid with a uniform thickness. The schematic of the two contacting surfaces and the textured micro-dimple is presented in Figure 1. The textured surface shows regular distribution of micro-dimple. For each dimple with a diameter $d$, depth $h$ is located in an imaginary square control unit with its length is $L$.

The following assumptions apply:

(1) The friction pair is in a full oil film lubrication state. That is, the surfaces of the friction pair are separated by a constant thickness of lubricating film, and the thickness is $h_{0}$. (2) Due to the thickness of the lubricating film $h_{0}$ is extremely small, it can be considered that the film pressure of the lubricating film does not change with the film thickness direction; (3) The lubricant is a Newtonian fluid, the volume force and the inertia force are neglected. The equation, with pressure control of the incompressible fluid under steady laminar flow conditions, can be obtained.

$$
\frac{\partial}{\partial x}\left(\frac{h^{3}}{6 \eta} \frac{\partial p}{\partial x}\right)+\frac{\partial}{\partial y}\left(\frac{h^{3}}{6 \eta} \frac{\partial p}{\partial y}\right)=U \frac{\partial h}{\partial x}
$$

Where $p$ is lubricating film pressure; $h$ is the film thickness; $\eta$ is fluid's dynamic viscosity; $U$ is the sliding velocity.

The two items on the left side of equation (1) represent the lubricant flow due to the pressure gradient, and the right one of the equal sign represents the lubricant flow due to the relative velocity. When the surface roughness is not considered, the thickness of the lubricating film at any point between the two sliding surfaces of the friction pair can be expressed by the following equations:

The thickness of film equation for an elliptical laser micro-dimple on the surface is

$h(x, y, t)=\left\{\begin{array}{lr}h_{0} & (x, y) \notin \Omega \\ h_{0}+h_{p}-\frac{h_{p}\left(x^{2}+y^{2}\right)}{r_{p}^{2}} & (x, y) \in \Omega\end{array}\right.$

The thickness of film equation for a spherical cap laser micro-dimple on the surface is

$$
h(x, y, t)=\left\{\begin{array}{lc}
h_{0} & (x, y) \notin \Omega \\
h_{0}+h_{p}-\left(r_{p}-\sqrt{r_{p}^{2}-\left(x^{2}+y^{2}\right)}\right) & (x, y) \in \Omega
\end{array}\right.
$$

The thickness of film equation for a conical laser micro-dimple on the surface is

$$
h(x, y, t)= \begin{cases}h_{0} & (x, y) \notin \Omega \\ h_{0}+h_{p}-\frac{h_{p} \sqrt{\left(x^{2}+y^{2}\right)}}{r_{p}} & (x, y) \in \Omega\end{cases}
$$

Where $\mathrm{x}$ and $\mathrm{y}$ are the Cartesian coordinates; $h(x, y, t)$ is the thickness of oil film at any point on the surface; $\Omega$ the region of all laser micro-dimples; ${ }^{h_{p}}$ is the depth of dimple; ${ }^{r_{p}}$ is the radius of dimple.

Considering that different surface morphology of micro-dimple could affect the hydrodynamic pressure of oil, three different kinds of morphologies for dimples are chosen to obtain the film pressure between the two contact surfaces. Reasonable boundary conditions must be set during the solution process. According to the formation theory of fluid lubrication film, the cavitation phenomenon does not cause the entire fluid film to cancel out the positive and negative pressures and lose the load carrying capacity. Therefore, the appropriate cavitation boundary conditions must be selected in the solution process to obtain a more approximate numerical solution. The film's load carrying capacity is calculated using Half-Sommerfeld boundary condition; specifically, the negative pressure of the obtained pressure distribution area will be considered as zero, and only the positive pressure will be used to calculate the average pressure.

Based on the above boundary conditions and combination of Eqs. (1) and (2), the film pressure of single micro-dimple with different surface morphologies between the two contact surfaces can be calculated and will be used to explain the mechanism responsible for reducing the friction with surface texturing in the next section.

\section{Numerical Calculation and Result Analysis}

In order to compare the oil film pressure change of micro-dimple structure with different morphologies, only the dynamic pressure changes of a single dimple are considered based on the mathematical models which are proposed in this paper. Assuming that the rotation speed of a diesel engine is $1000 \mathrm{R} / \mathrm{Min}$, the lubricating oil has a dynamic viscosity of $0.06 \mathrm{~Pa} . \mathrm{s}$; all the dimples with different morphologies have the same diameter of $40 \mathrm{um}$, and the depth of $20 \mathrm{um}$. The normal average thickness of the oil film on the sliding contact surface is set to $5 \mathrm{um}$. The pressure change of the oil film for each micro-dimple is chosen as an index to evaluate the effect of the hydrodynamic pressure lubrication on the textured surface. The results of calculation could 


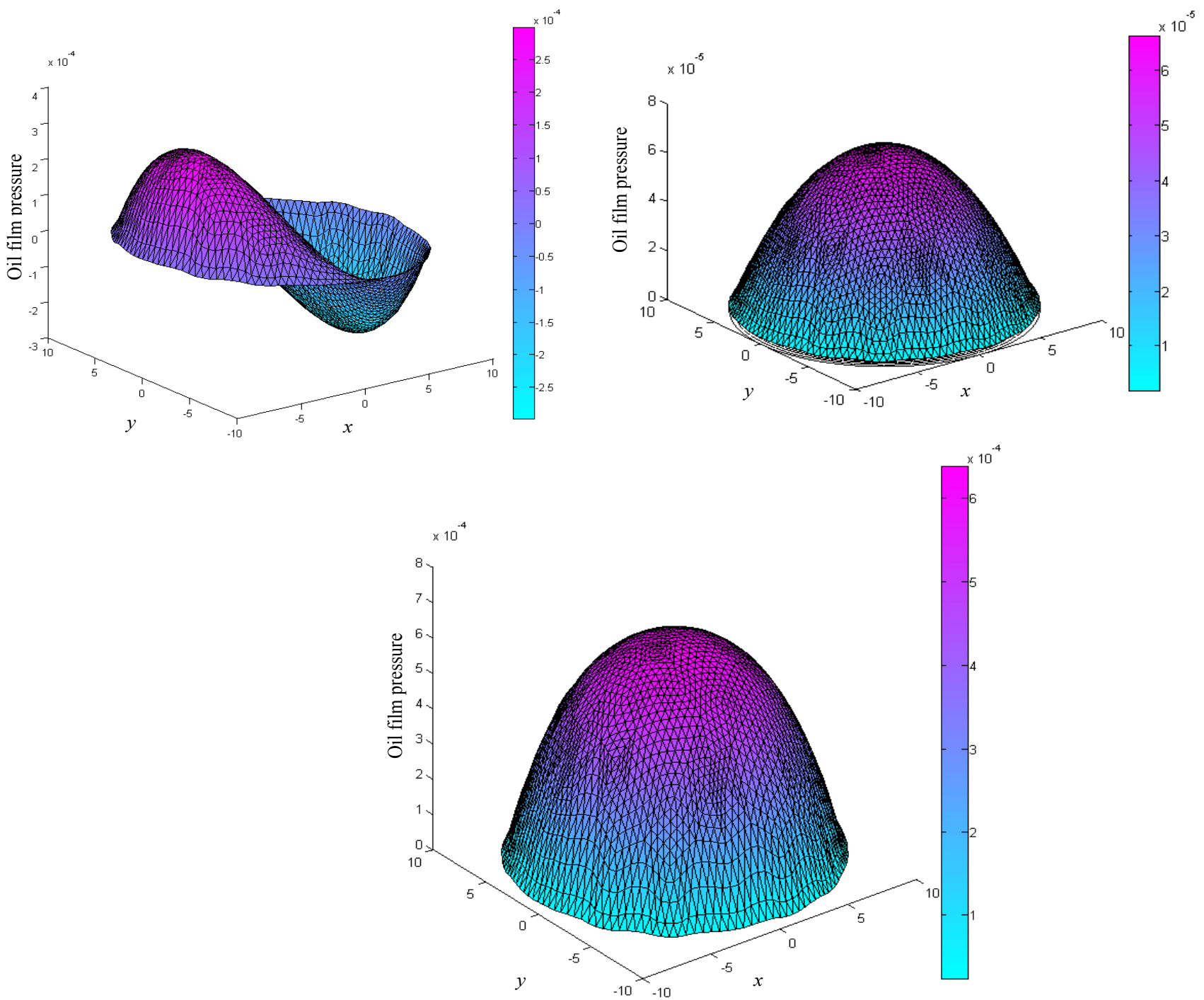

Figure 2: Schematic of oil film pressure for single dimple with different morphologies.

be used to compare the fact that which morphology structure of dimple is the better to achieve the aim of reducing friction. The Figure 2 shows the pressure distribution on the cross sections of elliptical dimple, conical dimple and spherical cap dimple, respectively. It can be seen that the pressure of elliptical dimple reaches a minimum at the inlet, and the pressure increases gradually until the maximum at the exit. It is shown that only the positive pressure zone, is formed in the micro-dimple with the shape appearance, which generate a positive pressure and provide a certain load capacity of oil film by the lubricating oil film in the elliptical micro-dimple. The spherical cap dimple has the highest hydrodynamic pressure and produces the oil film bearing capacity which is the largest. It can be seen from the Figure 2 that the spherical dimple has the best lubrication effect when analyzing the morphology of different single micro-dimple under the same conditions.

\section{Femtosecond Laser Ablation and Test}

Nowadays, micro scale machining based on the femtosecond laser is more and more applied in the field of research and engineering on a textured surface to achieve the purpose for reducing friction. It realizes that the micro-dimple morphologies are generated easily and effectively by femtosecond laser tool based on the results of numerical calculation, which becomes more and more important. In general, the shape of micro-dimple by the femtosecond laser ablation at a fixed focal position is mostly a tapered hole, which is a "V" shape. Therefore, it is difficult to machine a non-tapered structure on the surface of the sample by the fixed-point laser unless by using the moving scanning method to process the complicated structure of morphol- 


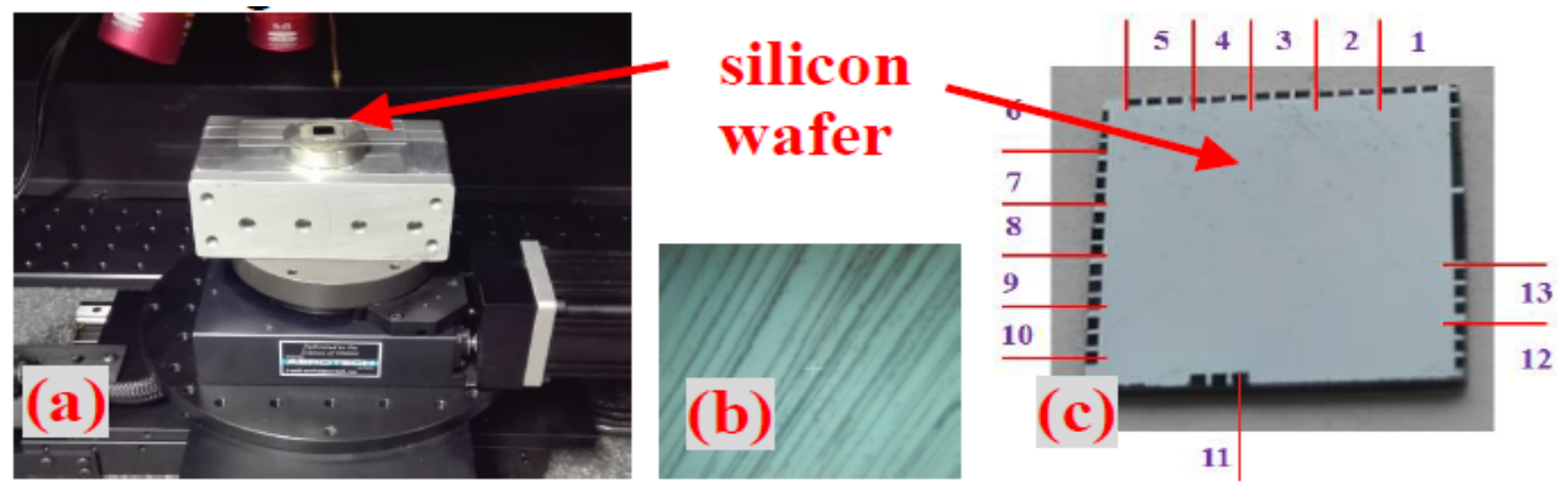

Figure 3: The machining center of femtosecond laser and ablated silicon wafer.

Table 1: The experimental data of laser ablation depth.

\begin{tabular}{|l|l|l|l|l|}
\hline Energy $(\mathbf{m W})$ & Step distance $(\mathbf{u m})$ & Scanning velocity $\mathbf{( m m} / \mathbf{s})$ & Scanning times & Ablation depth $(\mathbf{m m})$ \\
\hline 10 & 2 & 0.4 & 4 & 0.096 \\
\hline 10 & 2 & 1.2 & 6 & 0.062 \\
\hline 10 & 4 & 0.8 & 2 & 0.014 \\
\hline 10 & 6 & 0.4 & 4 & 0.083 \\
\hline 10 & 6 & 1.2 & 2 & 0.014 \\
\hline 30 & 2 & 0.8 & 4 & 0.113 \\
\hline 30 & 4 & 0.4 & 4 & 0.127 \\
\hline 30 & 4 & 1.2 & 10 & 0.096 \\
\hline 30 & 6 & 0.8 & 4 & 0.047 \\
\hline 40 & 4 & 2 & 2 & 0.056 \\
\hline 40 & 4 & 2 & 4 & 0.148 \\
\hline 40 & 4 & 2 & 6 & 0.198 \\
\hline 50 & 2 & 0.4 & 2 & 0.163 \\
\hline 50 & 2 & 1.2 & 2 & 0.061 \\
\hline 50 & 4 & 0.8 & 4 & 0.104 \\
\hline 50 & 6 & 0.4 & 4 & 0.173 \\
\hline 50 & 6 & 1.2 & 4 & 0.049 \\
\hline
\end{tabular}

ogy. Based on the previous study [19] of process attributes by femtosecond laser, more experiment researches were taken out further for the laws of ablation by the main laser parameters in the femtosecond laser machining center, as shown in Figure 3. The influence factors of shape and depth are studied by various combinations of the laser power, scanning velocity and step distance, which is concluded by some groups of microchannels were ablated under different ablation parameters. In summarizing of the results in Table 1 , the ablation depth is reduced with the increase of scanning velocity or the step distance and it increases with the laser power. The laser power and scanning velocity have significant influences on ablation depth and the step distance only presents its obvious effects on ablation depth when the laser power is high.

On the basis of mastering the laser parameters and their laws affecting the size and shape of the ablation process, precise control of the micromachining size can be achieved. For elliptical dimple, conical dimple and spherical cap dimple, the moving scanning method can be used to try to machine those. The moving scanning method is that the motion platform of femtosecond laser machining center continuously shifts the 


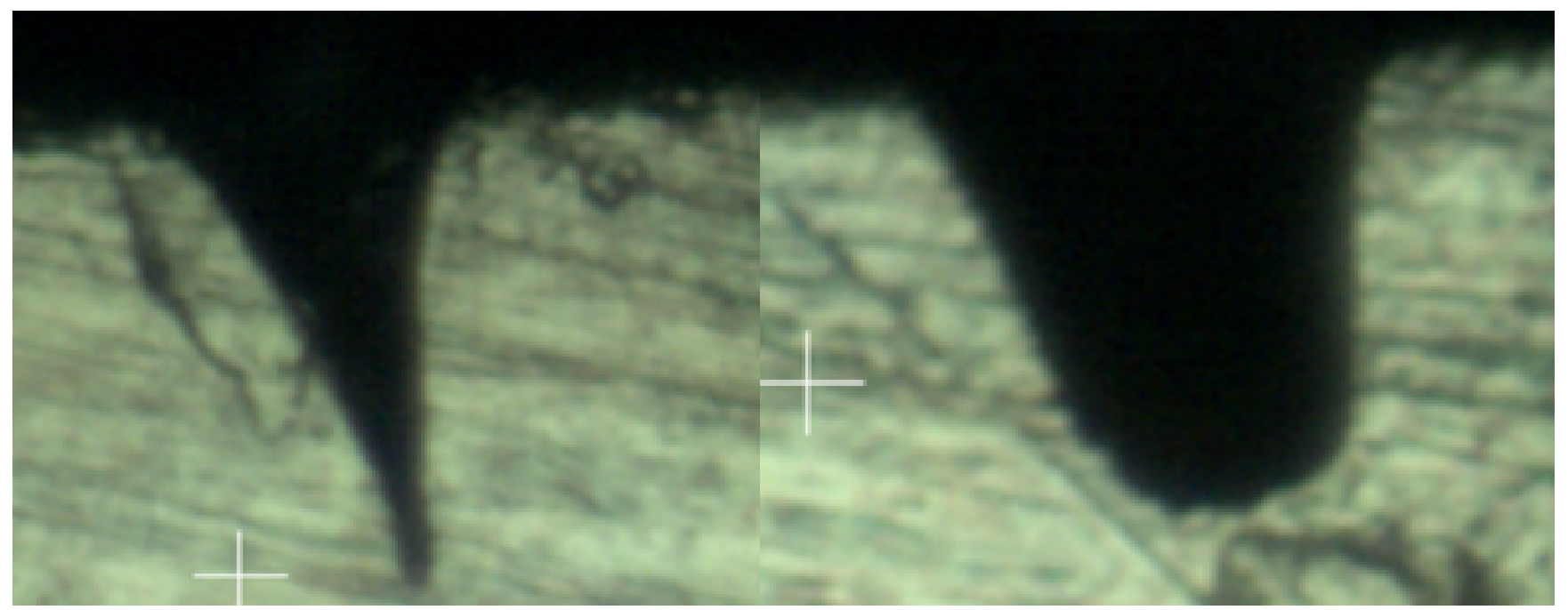

Figure 4: The schematic of "V" shaped dimple and spherical cap dimple on moving scanning method.

displacement during the processing according to a certain design path, and cooperates with different laser powers to complete the processing of various morphologies of dimples. The Figure 4 presents the processing schematic of "V" shaped dimple and spherical cap dimple on moving scanning method by the femtosecond laser. According to the moving scanning method, the regular arrays of spherical cap dimples were produced by femtosecond laser on the sample surface to verify the effect of reducing the friction of textured surface with spherical cap dimple in the friction test. The each dimple with a diameter of $100 \mathrm{um}$ and depth of 30 um was located on the sample surface, the area of arrays of dimples occupies the whole area of sample is $5 \%$. The friction tests were performed by using tribometer to compare the performance of reducing friction between the textured surface with arrays of dimple and non-textured surface. The results show that the textured samples have a lower coefficient of friction than the non-textured sample, and the friction coefficient with $5 \%$ distribution density of dimple is approximately $15 \%$ lower than that of the non-textured sample. The result is consistent with the conclusion of literature [20] on the friction coefficient with dimple textured structure is significantly less than that of non-textured surface.

\section{Conclusion}

A study was performed to investigate the viability of textured surface for improved tribological performance under fully lubricated conditions. The theoretical and simulation results of micro-dimple based on the Reynolds equation were developed. The results indicate that the surface texturing is important in reducing friction and the generation of hydrodynamic pressure is the responsible for reducing the friction with surface texturing. Among the three kinds of different morphologies of dimples, the spherical dimple has the best lubrication effect of hydrodynamic pressure In comparison to non-textured surfaces, the textured dimples were machined on the method of moving scanning by femtosecond laser, which displays the better hydrodynamic pressure in tests and the textured structure can reduce friction $15 \%$, is identified in the tests.

\section{Acknowledgements}

The authors acknowledge financial support from China Postdoctoral Science Foundation (2014M551380) and NSERC (Natural Sciences and Engineering Research Council of Canada) discovery grant (288157-2013).

\section{References}

1. IEtsion (2005) State of the art in laser surface texturing. Journal of Tribology 127: 248-253.

2. X Wang, K Kato, K Adachi, K Aizawa (2001) The effect of laser texturing of SiC surface on the critical load for transition of water lubrication mode from hydrodynamic to mixed. Tribology International 34: 703-711.

3. M Nakada (1994) Trends in engine technology and tribology. Tribology International 27: 3-8.

4. Hawkes CJ, Hardy GF (1936) Friction of piston rings. 
North East Coast Institution of Engineers and Shipbuilders, Newcastle upon Tyne, England.

5. Castleman Jr RA (1936) A hydrodynamical theory of piston ring lubrication. Journal of Applied Physics 7: 364-367.

6. Furuhama S (1959) A dynamic theory of piston-ring lubrication: $1^{\text {st }}$ report, calculation. Bulletin of JSME 2: 423-428.

7. Ruddy BL, Parsons B, Dowson D, Economou PN (1979) The influence of thermal distortion and wear of piston ring grooves upon the lubrication of piston rings in diesel engines. Proceedings of the $6^{\text {th }}$ Leeds-Lyon Symposium on Tribology.

8. Ruddy BL, Dowson D, Economou PN (1979) Piston ring lubrication: Part III-the influence of ring dynamics and ring twist. Energy Conservation through fluid film lubrication technology: Frontiers in research and design, 191-215.

9. Dowson D, Economou PN, Ruddy BL (1979) Piston ring lubrication part II. Theoretical analysis of a single ring and a complete ring pack. Energy conservation through fluid film lubrication technology: Frontiers in research and design, 23-52.

10.Wei Tang, Yuankai Zhou, Hua Zhu, Haifeng Yang (2013) The effect of surface texturing on reducing the friction and wear of steel under lubricated sliding contact. Applied Surface Science 273: 199-204.

11. Hamilton DB, Walowit JA, Allen CM (1966) A theory of lubrication by micro-irregularities. Journal of Basic Engineering 88: 177-185.

12.M Wakuda, Y Yamauchi, S Kanzaki, Y Yasuda (2003)
Effect of surface texturing on friction reduction between ceramic and steel materials under lubricated sliding contact. Wear 254: 356-363.

13.I Etsion (2004) Improving tribological performance of mechanical components by laser surface texturing. Tribology Letters 17: 733-737.

14.L Rapoport, A Moshkovich, V Perfilyev, A Gedanken, Y Koltypin, et al. (2009) Wear life and adhesion of solid lubricant films on laser textured steel surfaces. Wear 267: 1203-1207.

15.A Borghi, E Gualtieri, D Marchetto, L Moretti, S Valeri (2008) Tribological effects of surface texturing on nitriding steel for high-performance engine applications. Wear 265: 1046-1051.

16.G Ryk, I Etsion (2006) Testing piston rings with partial laser surface texturing for friction reduction. Wear 261: 792-796.

17.X Wang, K Adachi, K Otsuka, K Kato (2006) Optimization of the surface texture for silicon carbide sliding in water. Applied Surface Science 253: 1282-1286.

18.I Etsion, E Sher (2009) Improving fuel efficiency with laser surface textured piston rings. Tribology International 42: 542-547.

19.Zhang $P$, Chen L, Wang F, Tu P (2017) A high precision scheme for micro-channel processing in quartz glass using femtosecond laser. Microw Opt Technol Lett 59: 1993-2000.

20.Grabon W, Koszela W, Pawlus P, Ochwat S (2013) Improving tribological behaviour of piston ring-cylinder liner frictional pair by liner surface texturing. Tribology International 61: 102-108. 